\title{
HMM-BASED UNDERWATER TARGET CLASSIFICATION WITH SYNTHESIZED ACTIVE SONAR SIGNALS
}

\author{
Taehwan Kim, Keunsung Bae \\ School of Electrical Engineering and Computer Science, Kyungpook National University \\ 1370 Sankyukdong, Bukgu, 702-701, Daegu, Korea \\ phone: + 82-53-940-8627, fax: + 82-53-940-8827, email: onlyinkr@ee.knu.ac.kr \\ web: http://mir.knu.ac.kr
}

\begin{abstract}
This paper deals with underwater target classification using synthesized active sonar signals. Firstly, we synthesized active sonar returns from $3 D$ highlight model of underwater targets using the ray tracing algorithm. Then, we applied a multiaspect target classification scheme based on a hidden Markov model to classify them. For feature extraction from the synthesized sonar signals, a matching pursuit algorithm was used. Experimental results depending on the number of observations and signal-to-noise ratio are presented with our discussions.
\end{abstract}

\section{INTRODUCTION}

Underwater target detection and classification problems have been studied from many researchers for both military purposes and non-military ones. Various pattern recognition approaches with active sonar signals are under study, but there are many problems to be considered. The sonar signals are distorted in underwater environment, and the temporal and spectral characteristics of active sonar signals change in accordance with the aspect of the target even though it comes from the same one. In addition, since it is difficult to collect real data for research, most researchers focus on the experimentally generated data such as sonar returns from submerged elastic cylindrical shaped targets in the water tank or lake [1]-[3]. As an alternative approach to this, synthesized sonar signals on the certain target condition can be used. In that case conventional echo highlight model [4] could be used because of its simplicity.

HMM is a statistical signal processing technique to model non-stationary time sequences. In this model, a transition of state would occur when the statistical characteristics of a signal is changed in time. HMM has been widely used in pattern recognition areas such as speech, handwriting, gesture recognitions, and bioinformatics. In 1999, Paul Runkle et al introduced the HMM to multiaspect target recognition [5]-[6]. Each state of the HMM is characterized by the aspect of the target and a state transition occurs when the target aspect changes by the received sonar signals of different directions. In this paper, we applied the HMM-based multiaspect target classification method to the synthetic linear frequency modulated (LFM) sonar returns from highlight models of underwater targets.
Matching pursuit is a signal decomposition method that finds maximum correlations with basis vectors contained in an over-complete dictionary. It repeatedly calculates best matching coefficients for residual signals subtracted in the previous step. It has an important advantage to decompose a signal that any type of basis much correlated with the signal can be chosen for decomposition. In active sonar systems LFM signals or continuous wave signals are generally used. So a sinusoidal short pulse might be the best choice of basis when we use matching pursuit algorithm for feature extraction.

In this work, we synthesized active sonar returns based on ray tracing algorithm for 3D highlight models. We extracted feature vectors from each synthesized active sonar signal using matching pursuit algorithm [7]. Then we employed an HMM-based multiaspect target recognition scheme to classify each target correctly. Though the synthesized active sonar signals are in very limited conditions because we assumed very simple target models and environment, simulation results have shown that the given multiaspect HMM scheme is appropriate for underwater target classification.

This paper is organized as follows. The synthesis of active sonar signals from highlight models is described in the next section. In section 3, we briefly explain about matching pursuit based feature extraction from the synthesized sonar returns. The HMM-based multiaspect target classification method is explained in section 4 , and its experimental results with discussions are given in section 5. Finally, conclusion is given in section 6 .

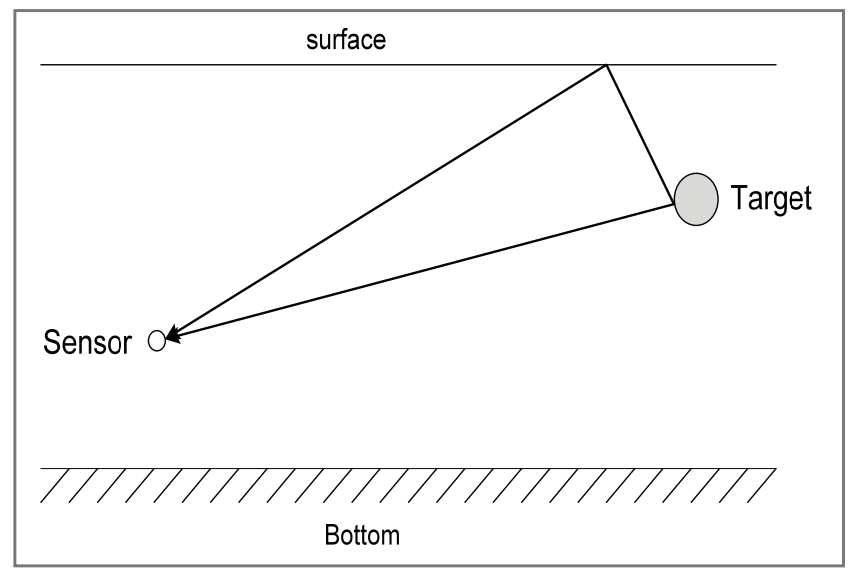

Figure 1 - Environment for synthesis of active sonar target signals. 


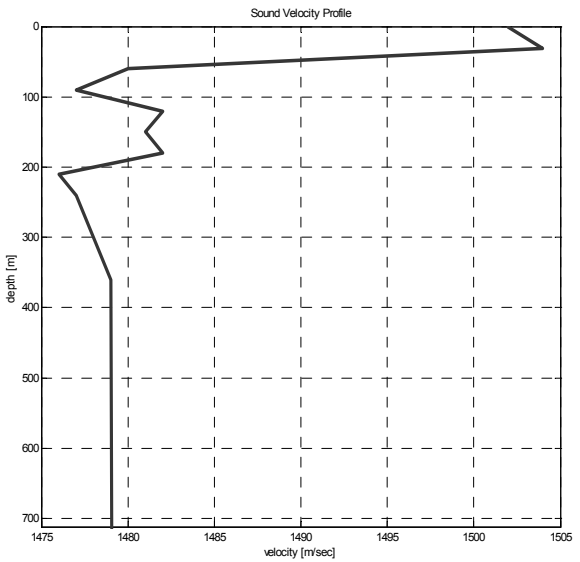

Figure 2 - The sound velocity profile

\section{SYNTHESIS OF ACTIVE SONAR RETURNS USING HIGHLIGHT MODELS}

For the synthesis of active sonar signals, we assumed a simple environment of active sonar returns as shown in Figure 1. We considered the direct reflections as well as indirect reflections from sea level and bottom level. The depth of water is set to $300 \mathrm{~m}$, the transmitter and receiver are located at the same position of the sea level, i.e., monostatic mode, and an unknown target is at $50 \mathrm{~m}$ below the sea level. We adopted the sound velocity profile to calculate the sound velocity at a certain depth of water as given in Figure 2. Figure 3 shows 4 types of highlight models of underwater targets designed for synthesis of sonar returns. All the targets have several highlights mainly lying in the horizontal line. Each highlight reflects acoustic wave in all directions. Underwater acoustic wave is then propagated over being attenuated and bent by sound velocity.

We can obtain the synthesized signal by summing traced signals from each highlight at the receiver position. In this work, we generated active sonar returns for each target by varying its aspect from 0 to 360 degrees by 1 degree increment. Figure 4 shows examples of synthesized active sonar signals from the target (d) in Figure 3 . Three segments of the signal shown in 60 and 90 degrees of aspect might be due to three paths of reflection (direct, surface reflection, and bottom reflection) that we considered when synthesizing.

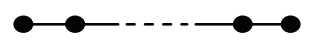

(a)

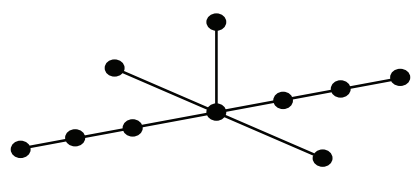

(c)

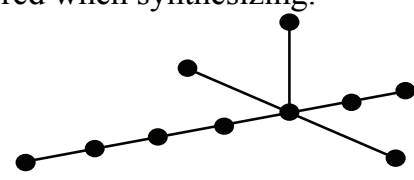

(b)

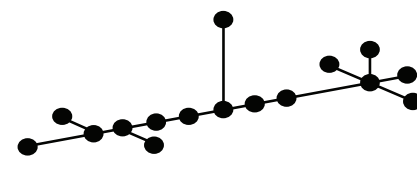

(d)
Figure 3-3D highlight models of targets for synthesis of active sonar signals.
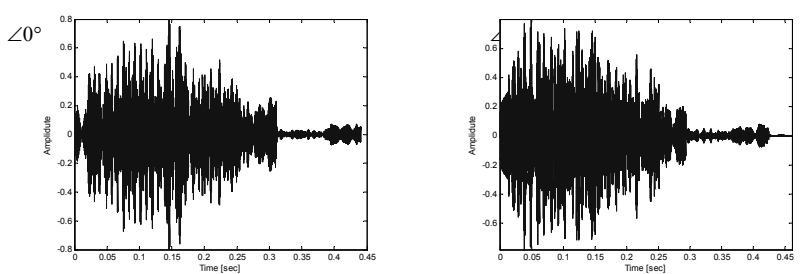

$\angle 60^{\circ}$
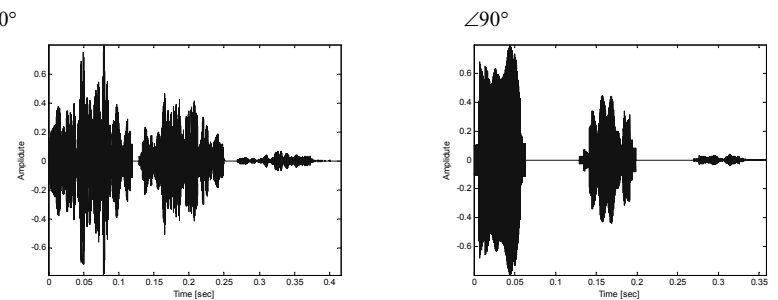

Figure 4 - Examples of generated active sonar signals from target (d) in Figure 3.

\section{FEATURE EXTRACTION USING MATCHING PURSUIT ALGORITHM}

Matching pursuit is a recursive signal decomposition method used for finding the maximum correlations with basis vectors from multi-dimensional data using an over-complete dictionary. Here dictionary denotes the list of basis functions used for analysis. Matching pursuit is widely used for sparse coding, compression, and feature extraction. It has a powerful advantage in choosing appropriate basis compared with other signal processing techniques such as Fourier transform or wavelet transform. It can choose any wave that has much correlation with analysing signals. In this work, we used a chirp-based dictionary, since the synthesized active signals are supposed to be transmitted using the LFM signals.

Firstly, an arbitrary signal is projected onto the dictionary using matching pursuit. From the first iteration, we are able to get the coefficient as described in equations (1) and (2).

$$
\begin{aligned}
& f(t)=\left\langle f, w_{0}\right\rangle w_{0}(t)+R_{1}(t) \\
& \left\langle f, w_{k}\right\rangle=\int f(t) w_{k}(t) d t
\end{aligned}
$$

Here $w_{0}(t)$ is a first basis selected by having highest correlation coefficient on the dictionary and $R_{1}(t)$ is a residual signal after first iteration of matching pursuit process. Second matching pursuit iteration is processed on the residual obtained from the first iteration. After $L$-th iteration a signal is decomposed as shown in equation (3).

$f(t)=\sum_{l=0}^{L-1}\left\langle R_{l}, w_{l}\right\rangle w_{l}(t)+R_{L}(t), \quad R_{0} \equiv f(t)$

Since we assumed that the LFM signals were transmitted from the sensor to synthesize active sonar returns, we used the chirp-based dictionary given in equation (4). 


$$
\begin{aligned}
& x_{A, f_{0}, k, \tau, \phi}(t)= \\
& \quad A \sin \left(2 \pi\left(f_{0}+\frac{k}{2}(t-\tau)\right)(t-\tau)+\phi\right) \operatorname{Rect}(t-T / 2-\tau)
\end{aligned}
$$

Here, $f_{0}$ denotes the center frequency of a chirp signal, $k$ is a chirp rate, $\tau$ is a time delay, and $\phi$ is phase, and all parameters can be extracted during iterations. And $T$ is the width of rectangular window applied in process. Since $f_{0}$ and $k$ of the sonar return have little information about the target, in our work, we used the time delay parameters obtained after 15 iteration processes as a feature vector, and is given like equation (5).

$$
\begin{aligned}
& \mathbf{y}=\left[\tau_{2}-\tau_{1}, \tau_{3}-\tau_{1}, \ldots, \tau_{15}-\tau_{1}\right] \\
& \text { where } \tau_{1} \leq \tau_{2} \leq \cdots \leq \tau_{15}
\end{aligned}
$$

\section{HIDDEN MARKOV MODEL-BASED MULTI- ASPECT TARGET CLASSIFICATION}

\subsection{Multiaspect HMM model}

In a multiaspect HMM-based classification method, a state is characterized by aspect angle interval of a target and a transition of state occurs when statistical characteristics of aspect angle interval are significantly changed. Each aspect angle interval is modelled by mixtures of Gaussian distribution. In our research we applied it for underwater target classification with synthesized active sonar signals. In the model 0 90 degrees of the target aspect is divided into five states having different characteristics as shown in Figure 5-(a) [5]. Because targets have almost symmetric shape, other aspect of a target can share the states defined above. Fig. 5-(b) shows a modified ergodic HMM used in this work. In this model, a state transition is allowed only from one to adjacent state except at state 1 and 5. At these states, a transition is restricted to only one state, i.e., state 2 and 4 , respectively.

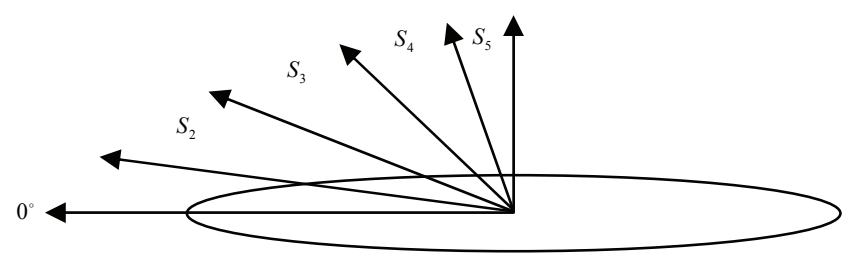

(a)

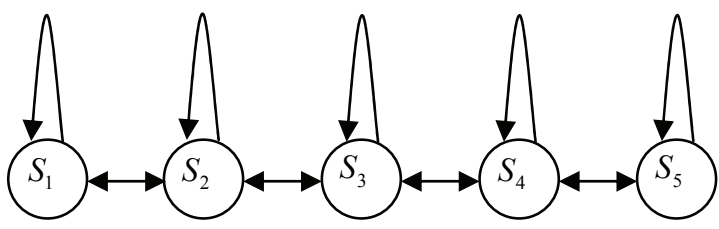

(b)

Figure 5 - Schematics of multiaspect HMM

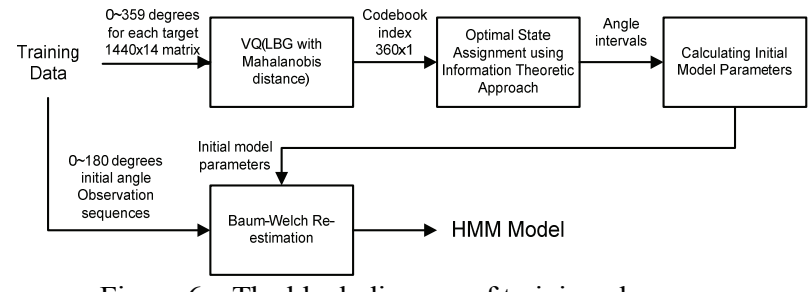

Figure 6 - The block diagram of training phase.

\subsection{HMM model training phase}

Fig. 6 shows the block diagram of training phase of a multiaspect HMM. To assign initial angle interval to each state, we generated a codebook using vector quantization from all feature vectors having aspect from 0 to 360 degrees. Then optimal initial state assignment is carried out based on information theoretic approach. That is, we minimized the conditional entropy of equation (6) using a recursive approach.

$$
\begin{aligned}
& H(q \mid v)= \\
& \quad-\sum_{n=1}^{N} \sum_{i=1}^{I} P\left(q=s_{n}, v=i\right) \cdot \log _{2} P\left(q=s_{n} \mid v=i\right)
\end{aligned}
$$

Here $H(\cdot \mid \cdot)$ denotes conditional entropy, $N$ is the number of states, and $I$ is the size of a codebook. From this we can calculate initial state intervals, $\varphi_{1}, \ldots, \varphi_{N}$, with the following equations (7).

$$
\pi_{n}=\frac{\varphi_{n}}{\sum_{n=1}^{N} \varphi_{n}}
$$

$a_{n n}=p\left(q_{k+1}=s_{n} \mid q_{k}=s_{n}\right)=\frac{\varphi_{n}-\delta \theta}{\varphi_{n}}$

$a_{n m}=p\left(q_{k+1}=s_{m} \mid q_{k}=s_{n}\right)=\frac{\delta \theta}{2 \varphi_{n}}, \quad|n-m|=1$

$a_{n m}=0, \quad|n-m|>1$

$b_{i n}=p\left(\mathbf{y}_{m} \mid q_{k}=s_{n}, T_{k}\right)=\sum_{l=1}^{L} w_{l} g_{l}\left(\mathbf{y}_{m} \mid q_{k}=s_{n}, T_{k}\right), \sum_{l=1}^{L} w_{l}=1$

Then we reiterate each initial HMM model using the BaumWelch re-estimation until the model is converged.

\section{EXPERIMENTAL RESULTS}

In the synthesis of active sonar signals, we set the sampling frequency to $31.25 \mathrm{kHz}$, and LFM pulse duration to $50 \mathrm{~ms}$. The center frequency and bandwidth of the LFM signal are $7 \mathrm{kHz}$, and $400 \mathrm{~Hz}$, respectively. For training each HMM model, 181 observation sequences having initial aspect from 0 to 180 degrees were used and for testing 361 observation sequences having initial aspect from 0 to 360 degrees were used. Each observation sequence has 10 concatenated feature vectors with 5 degree interval. We investigated classification performance by varying the number of observations from 1 to 10 . Matching pursuit feature extraction method was employed with MPTK[8] and all recognition results were obtained using the HMM toolbox, i.e., HTK[9]. 


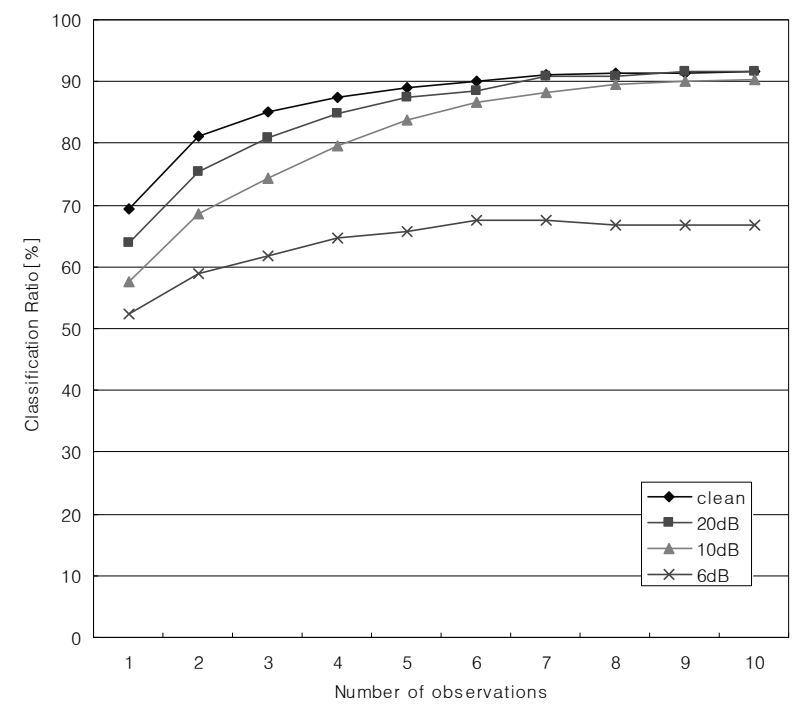

Figure 7 - The classification results under noisy environment.

Figure 7 shows the classification results depending on the number of observations as well as signal-to-noise ratio (SNR). In principle, correct classification rate decreases as the number of observation decreases as well as SNR. From the analysis of the above results with the HTK output file, we found that most classification errors occurred at target (b) in Figure 3 when the aspect is 180 to 360 degrees. We think this result comes out because the target 2 is asymmetric unlike others, and further study is under way to verify it. Figure 8 shows the classification results depending on angle interval variation of the observation sequences. It shows that the HMM-based recognition is robust against some variations in the aspect angle interval. This is an advantage of an HMM-based recognition system. On the contrary to this, we found that the HMM recognition system should have a sufficient length of observation sequences for train and recognition compared to other pattern recognition algorithms.

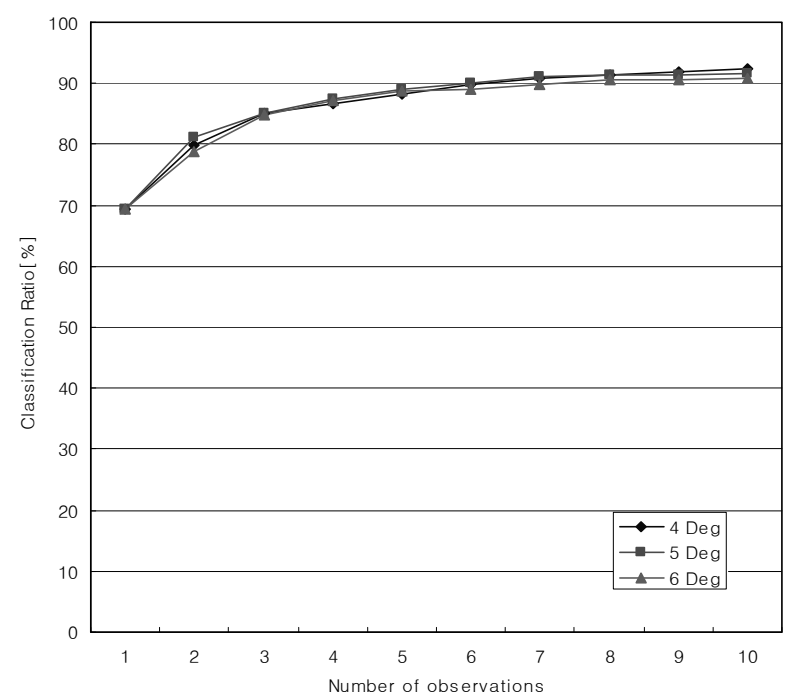

Figure 8 - The classification results according to aspect angle interval of the observation sequences.

\section{CONCLUSION}

In this paper, we synthesized active sonar signals using target highlight models based on ray tracing algorithm. Then multiaspect HMM-based target classification experiments were carried out by varying the number of observations and aspect angle interval of observation sequences. Experimental results have shown that the method in this study works quite well even if we do not know the aspect information of the target. Further research will be focused on the modelling the HMM more accurately to improve the recognition performance as well as synthesizing active sonar returns with a more elaborate model of a target.

\section{ACKNOWLEDGEMENT}

This work was supported by Defense Acquisition Program Administration and Agency of Defense Development under the contract UD100002KD.

\section{REFERENCES}

[1] A. Pezeshki, M. R. Azimi-Sadjadi, and L. L. Scharf, "Undersea Target Classification Using Canonical Correlation Analysis," IEEE Journals of Oceanic Engineering, vol. 32, no. 4, pp. 948-955, Oct. 2007.

[2] M. R. Robinson, M. R. Azimi-Sadjadi, and J. Salazar, "Multi-Aspect Target Discrimination Using Hidden Markov Models and Neural Networks," IEEE Transactions on Neural Networks, vol. 16, no. 2, pp. 447-459, Mar. 2005.

[3] M. R. Azimi-Sadjadi, D. Yao, A. A. Jamshidi, and G. J. Dobeck, "Underwater Target Classification in Changing Environments Using an Adaptive Feature Mapping," IEEE Transactions on Neural Networks, vol. 13, no. 5, pp. 10991111, Sep. 2002.

[4] B. Kim, H. Lee, and M. Park, "A Study on Highlight Distribution for Underwater Simulated Target," Proceeding of ISIE 2001, vol. 3, pp. 1988-1992, 2001.

[5] P. R. Runkle, P. K. Bharadwaj, L. Couchman, and L. Carin, "Hidden Markov Models for Multi-aspect Target Classification, IEEE Transactions on Signal Processing, vol. 47, no. 7, pp. 2035-2040, 1999.

[6] P. R. Runkle, L. Carin, L. Couchman, T. J. Yoder, and J. A. Bucaro, "Multi-aspect Target Identification with Wave-Based Matched Pursuits and Continuous Hidden Markov Models," IEEE Transactions on Pattern Analysis and Machine Intelligence, vol. 21, no. 12, 1999.

[7] S. G. Mallat and Z. Zhang, "Matching Pursuits With Time-Frequency Dictionaries," IEEE Transaction on Signal Processing, vol. 41, no. 12, pp. 3397-3415, Dec. 1993.

[8] Matching Pursuit Toolkit, http://mptk.irisa.jr/

[9] HMM Toolkit, http://htk.eng.cam.ac.uk/ 